Check for updates

Cite this: RSC Adv., 2019, 9, 26024

\title{
Strain engineering on the electronic states of two- dimensional GaN/graphene heterostructure
}

\author{
Zhongxun Deng (D) ab and Xianhui Wang*a \\ Combining two different layered structures to form a van der Waals (vdW) heterostructure has \\ recently emerged as an intriguing way of designing electronic and optoelectronic devices. Effects \\ of the strain on the electronic properties of GaN/graphene heterostructure are investigated by \\ using first-principles calculation. In the GaN/graphene heterostructure, the strain can control not \\ only the Schottky barrier, but also contact types at the interface. Moreover, when the uniaxial strain \\ is above $-1 \%$ or the biaxial strain is above $0 \%$, the contact type transforms to ohmic contact. These \\ results provide a detailed understanding of the interfacial properties of GaN/graphene and help to \\ predict the performance of the GaN/graphene heterostructure on nanoelectronics and \\ nanocomposites.
}

Received 29th April 2019

Accepted 5th August 2019

DOI: 10.1039/c9ra03175h

rsc.li/rsc-advances

been directly synthesized experimentally via graphene encapsulation. ${ }^{27}$ Thus, GaN/graphene heterostructure form in this process and the properties of GaN are sensitive to the interaction between GaN and graphene. Nevertheless, the fundamental electronic properties of GaN/graphene heterostructure remain unexplored.

In this work, we study the electronic properties of GaN/ graphene heterostructure by first-principles calculations. The intrinsic properties of the GaN and graphene layers are preserved in the weak vdW heterostructure at equilibrium interfacial distance. Moreover, the strain can control not only the Schottky barrier, but also contact types at the interface in the GaN/graphene heterostructure. These results provide a detailed understanding of the interfacial properties of GaN/ graphene and help to predict the performance of the GaN/ graphene heterostructure on nanoelectronics and nanocomposites.

thus leading to some very intriguing phenomena such Hofstadter's butterfly spectrum, ${ }^{17,18}$ strongly bound excitons, ${ }^{19,20}$ and spin valley polarization. ${ }^{21,22}$

Gallium nitride (GaN) is an important commercial semiconductor for optoelectronic applications in the visible and near-ultraviolet parts of the spectrum. ${ }^{23,24}$ Devices employing atomically thin GaN quantum wells and tunnel injection achieve deep-UV light emission in a desirable spectral range for sterilization applications. ${ }^{25,26}$ Unlike other 2D materials, such as graphene or TMDs, bulk GaN crystallizes with the nonlayered wurtzite structure and is not expected to adopt a 2D form with traditional exfoliation methods. However, 2D GaN has recently

${ }^{a}$ Shanxi Province Key Laboratory of Electrical Materials and Infiltration Technology, School of Materials Science and Engineering, Xi'an University of Technology, Xi'an 710048, Shaanxi, P. R. China.E-mail:xhwang693@xaut.edu.cn

${ }^{b}$ Energy and Engineering College, Yulin University, Yulin 719000, Shaanxi, P. R. China

\section{Computational method}

This article uses density functional theory (DFT) with the projected augmented wave (PAW) method, as implemented within the Vienna $A b$ initio Simulation Package (VASP). ${ }^{28-31}$ The generalized gradient approximation is utilized as the exchangecorrelation functional, which can reasonably predict the band gap of GaN. ${ }^{32}$ The vdW correction proposed by Grimme is included in our calculations due to its good description of longrange vdW interactions. ${ }^{33}$ the heterostructure calculations are performed by using the $5 \times 5 \times 1$ and $13 \times 13 \times 1$ MonkhorstPack $K$-point mesh for relaxation and electron static calculations, respectively. The energy cutoff for plane-wave expansion was set to $450 \mathrm{eV}$. A large vacuum space of $20 \AA$ was added to avoid interaction between adjacent images. All atoms are 
allowed to be fully relaxed until the atomic Hellmann-Feynman forces are smaller than $0.01 \mathrm{eV} \AA^{-1}$ and the convergence criterion of energy in the self-consistency process is set to $10^{-5} \mathrm{eV}$. Visualizations of supercells and structures were done with the software VESTA. ${ }^{34}$

The binding energy at the interface is defined as

$$
E_{\mathrm{b}}=\left[E_{\mathrm{GaN} / \text { graphene }}-\left(E_{\mathrm{GaN}}+E_{\text {graphene }}\right)\right] / N_{\mathrm{C}},
$$

where $E_{\mathrm{b}}$ is the binding energy; $E_{\mathrm{GaN} / \text { graphene }}, E_{\mathrm{GaN}}$, and $E_{\text {graphene }}$ are the total energy of the GaN/graphene heterostructure, GaN monolayer, and graphene monolayer, respectively; and $N_{\mathrm{C}}$ is the number of carbon atoms in the supercell. The system is stable for negative $E_{\mathrm{b}}$ and, the smaller the $E_{\mathrm{b}}$ value is, the stronger the heterostructure binding is. To gain further insight into the bonding nature and interlayer interaction, the plane-averaged charge density difference $\Delta \rho$ is calculated:

$$
\Delta \rho=\rho_{\mathrm{GaN} / \text { graphene }}-\rho_{\mathrm{GaN}}-\rho_{\text {graphene }},
$$

in which $\rho_{\mathrm{GaN} / \text { graphene }}, \rho_{\mathrm{GaN}}$, and $\rho_{\text {graphene }}$ are the plane-averaged charge densities of the GaN/graphene heterostructure, GaN and graphene, respectively.

\section{Results and discussion}

In order to study the hybrid vdW heterostructure of GaN and graphene, the structural parameters of 2D monolayer GaN and graphene are calculated firstly. After the total energy relaxation, the optimized lattice constants of graphene and GaN are $2.469 \AA$ (Fig. 1a) and $3.253 \AA$ (Fig. 1b), respectively. These results are consistent with previous studies. ${ }^{35,36}$ The supercell of the heterostructure is composed of $3 \times 3$ supercell of GaN and $4 \times 4$ supercell of graphene, as shown in Fig. 1c. In our models, we fix the lattice constant of the heterostructures to the value of GaN and change the lattice constant of graphene to adjust to those of GaN. There is a lattice mismatch of $1.2 \%$ compressive strain for graphene, which is too small and no significant changes on the electronic properties in the heterostructure.

The equilibrium structure is yielded by applying the two-step optimization on the designed lattice. For step one, the interlayer distance is changed and the binding energy was calculated, as well as the results were fitted into the well-known Buckingham potential equation, as shown below

$$
E_{\mathrm{b}}=A e^{-B d}-\frac{C}{d^{6}},
$$

in which $A=-7.492, B=1.158$, and $C=-136.063$ are fitting parameters, $d$ is the interlayer distance, and $E_{\mathrm{b}}$ represents the binding energy. The binding energy as a function of the interlayer distance and fitting curve is shown in Fig. 1d. The predicted equilibrium interlayer distance read from the fitting curve is about $3.308 \AA$. for step two, a structure with an interlayer distance of $3.308 \AA$ is constructed and placed into a full relaxation to yield the final equilibrium structure. The interlayer distance between the monolayer GaN and graphene of the fully optimized structure is $3.315 \AA$, with a binding energy of $-59 \mathrm{meV}$ per $\mathrm{C}$ atom, which is of the same order of magnitude as that of other heterostructures such as $\mathrm{MoS}_{2} /$ graphene, ${ }^{37}$ black phosphorene/graphene, ${ }^{38-41}$ and arsenene/graphene. ${ }^{42,43}$ According to the negligible binding energy, GaN is found to interact very weakly with graphene, suggesting that $\mathrm{GaN}$ is bound to graphene via $\mathrm{vdW}$ interactions.

Although GaN and graphene are bonded by weak vdW forces, the interlayer interaction exhibits influence on the electronic properties of GaN/graphene heterostructure. The calculated electronic states of the GaN/graphene heterostructure at the

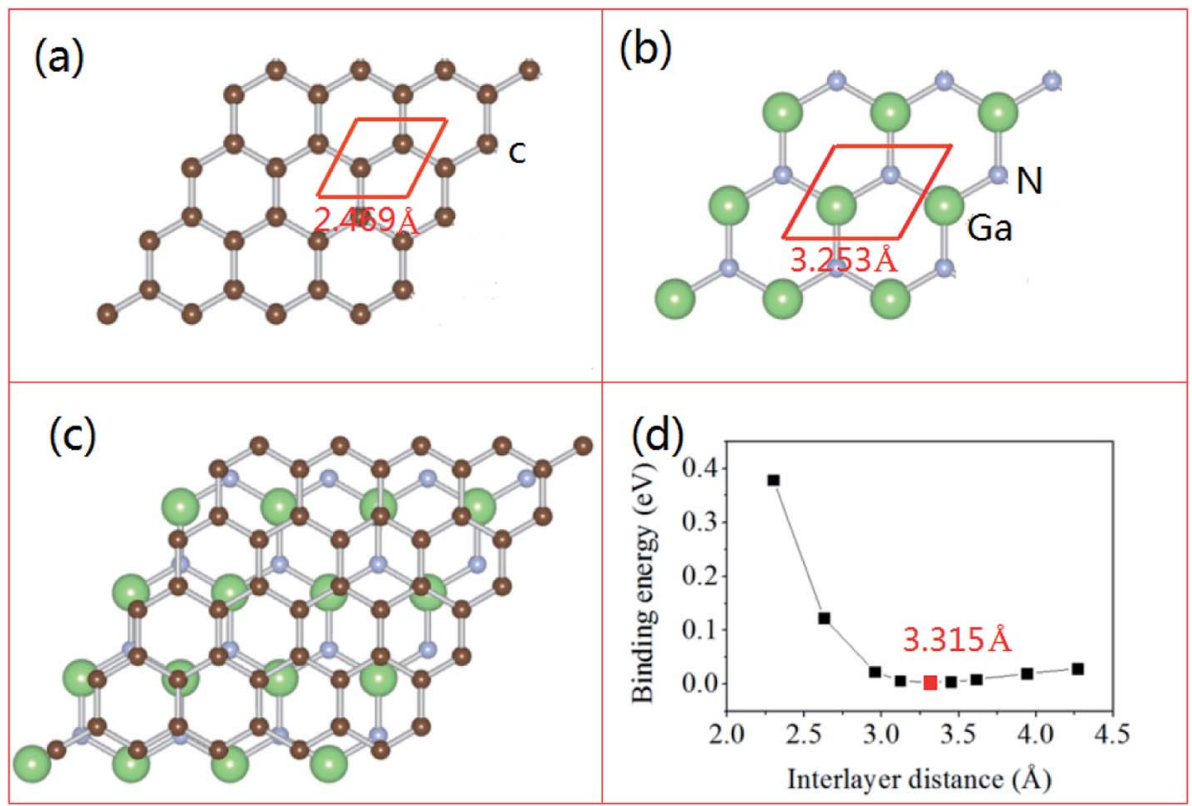

Fig. 1 Schematic illustration of the crystal structures of monolayer (a) graphene and (b) GaN, and (c) GaN/graphene heterostructure. (d) Binding energy of GaN/graphene heterostructure as a function of the interlayer distance. 


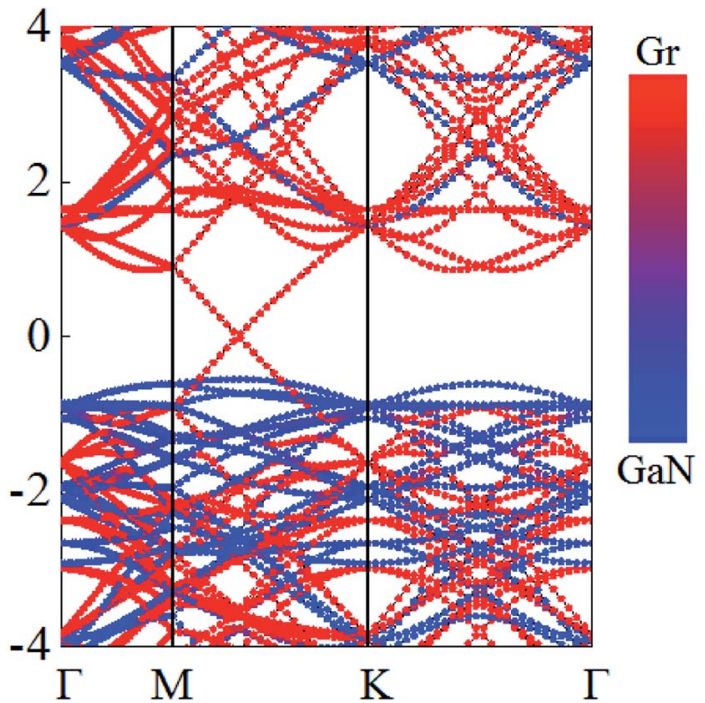

Fig. 2 Band structure of GaN/graphene heterostructure with an interlayer distance of $3.315 \AA$.

equilibrium interlayer distance are displayed in Fig. 2. From Fig. 2, it is evident that both the Dirac cone of graphene and the semiconducting nature of $\mathrm{g}$-GaN are quite well preserved. Linear dispersion characteristic of graphene is retained in bands near the Fermi level. Besides, the Fermi level is located exactly at the Dirac cone, demonstrating that the effects of the interaction and charge transfer are vanishingly small. Compared with band gap of $2.20 \mathrm{eV}$ for the free standing $\mathrm{GaN},{ }^{44,45}$ the band gap of $2.164 \mathrm{eV}$ for the GaN sublayer was almost unchanged up contacting with graphene. Besides, the valence band maximum of the GaN sublayer is low to the Fermi level. These results suggest that graphene can be a very promising material for the metal electrode, and it can enhance the contact performance while still maintaining the electronic properties of GaN.

The interlayer interaction between GaN and graphene is through the charge redistribution in the heterostructure. In order to investigate the effects of charge transfer on the electronic properties of $\mathrm{GaN}$ on graphene, the planar-averaged the plane-averaged electrostatic potentials of monolayer GaN, graphene and GaN/graphene heterostructure along $z$-direction are calculated, as depicted in Fig. 3a. The work function $W$ is defined as: $W=E_{\mathrm{vac}}-E_{\mathrm{F}}$, where $E_{\mathrm{vac}}$ and $E_{\mathrm{F}}$ are the vacuum energy and Fermi energy, respectively. The work function of the GaN/graphene heterostructure (4.935 eV) is smaller than that of the monolayer GaN $(5.267 \mathrm{eV})$, but larger than that of the monolayer graphene $(4.336 \mathrm{eV}){ }^{46}$ The difference in work function between the monolayer GaN and graphene indicated that the electrons flowed from graphene to GaN until the Fermi energy of two sublayers was aligned. Fig. $3 \mathrm{~b}$ illustrates the isosurface of the charge difference for GaN/graphene heterostructure, where the yellow and cyan isosurfaces represent gain and depletion of charge density. The change at interfaces indicates that the electrons transfer from graphene side to GaN side, where the holes remain in graphene side. Net charge accumulation leads to the formation of the built-in electric field at the interface should exhibit strong effects on the carrier dynamics and improve efficiency for charge injection.

Then, the electronic properties of the GaN/graphene heterostructure are modulated when the uniaxial ( $x$-direction) and biaxial ( $x$ - and $y$-direction) strain are applied. For uniaxial strain, the band gap is reduction with increasing applied strain (both tensile and compressive). As shown in Fig. 4, the band gaps are 1.930, 1.928, 1.900 and $1.814 \mathrm{eV}$ when the uniaxial strains are $-2 \%,-4 \%,-8 \%$ and $-10 \%$. It is also found that the Fermi level shifts to near the valence band maximum and away from the conduction band minimum, but the difference between Fermi level and the conduction band minimum maintains larger than that between Fermi level and the valence band maximum. When the uniaxial strains are $2 \%, 4 \%$ and $8 \%$, the band gaps of GaN/graphene heterostructure are 1.915, 1.724 and $1.273 \mathrm{eV}$. Although the Fermi level shifts to near the conduction band minimum, the difference between Fermi level and the valence band maximum is almost not change with increasing applied the uniaxial tensile strain.

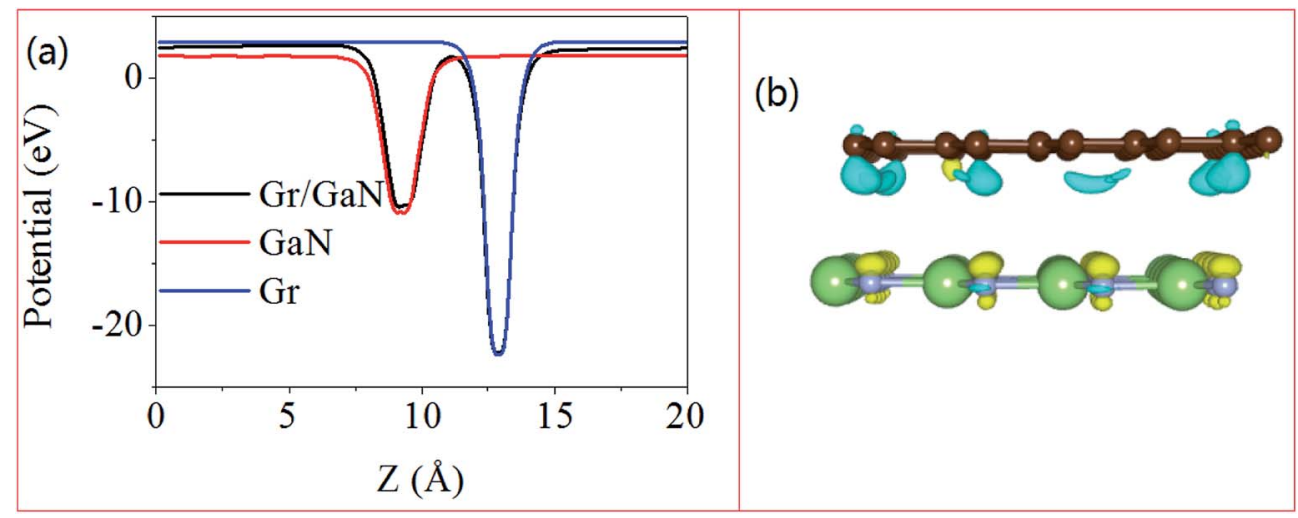

Fig. 3 (a) Electrostatic potentials of monolayer graphene and GaN, and GaN/graphene heterostructure in the $z$ direction are denoted by black, red, and blue lines, respectively. (b) Isosurface of the charge difference for GaN/graphene heterostructure. The isosurface of charge difference with a value of 0.0008 e $\AA^{-3}$. The yellow and cyan isosurfaces represent gain and depletion of electrons, respectively. 

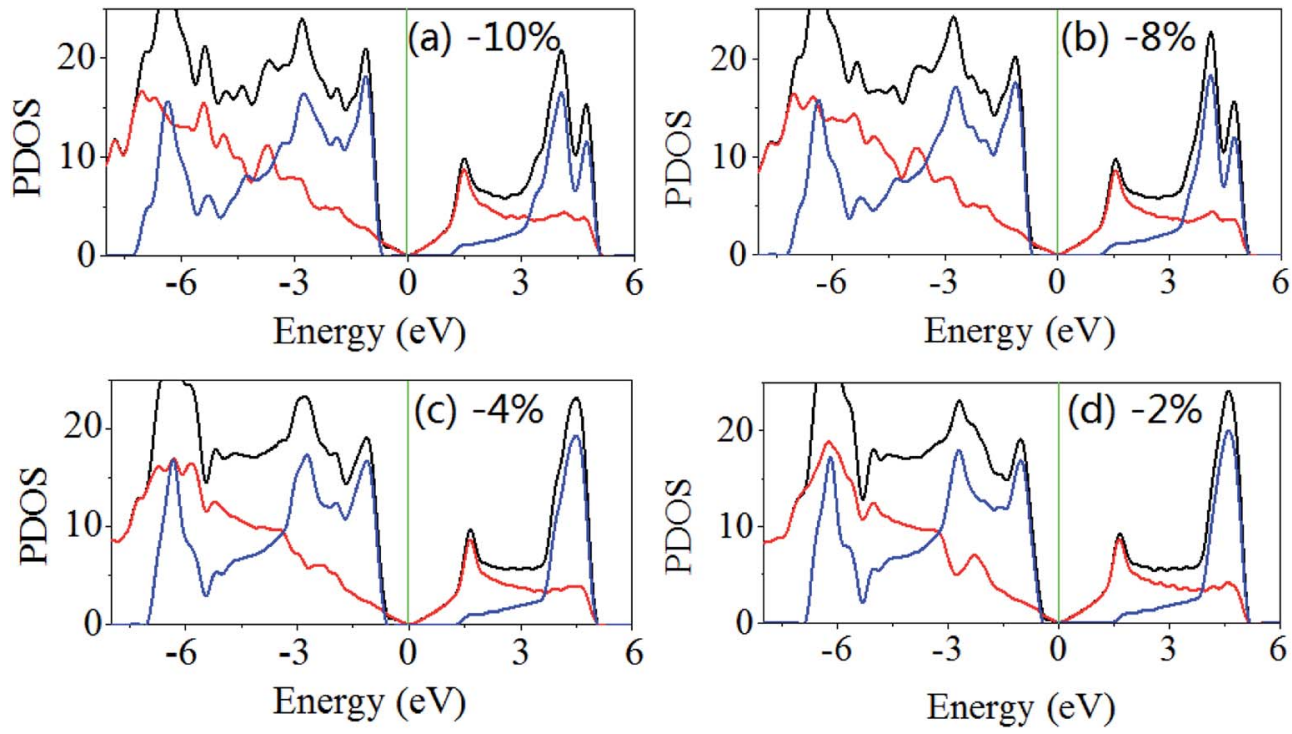

Fig. 4 Density of states the GaN/graphene heterostructure with different applied uniaxial strain along $x$-direction. The negative value represents the compressive strain.
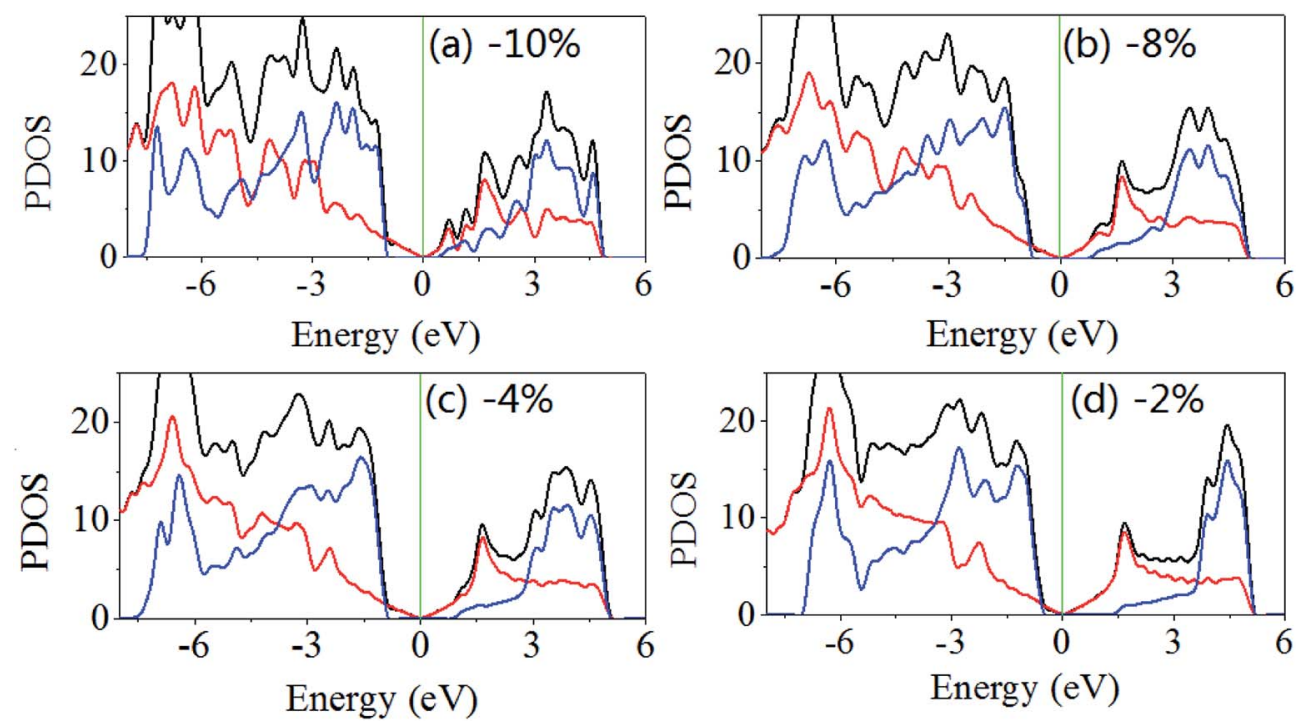

Fig. 5 Density of states of GaN/graphene heterostructure with different applied biaxial strain in-plane ( $x$ and $y$, equally). The negative value represents the compressive strain.

The same results are obtained when the biaxial strain is applied. As shown in Fig. 5, when the biaxial strains are $-2 \%$, $-4 \%,-8 \%$ and $-10 \%$, the band gaps are $1.896,1.843,1.528$ and $1.384 \mathrm{eV}$. The Fermi level shifts to near the valence band maximum and away from the conduction band minimum, and when the biaxial strain less than $8 \%$ the difference between Fermi level and the valence band maximum is large than that between Fermi level and the conduction band minimum. When increasing applied the biaxial tensile strain, the difference between Fermi level and the conduction band minimum decreases rapidly but the difference between Fermi level and the valence band maximum changes slightly.
To quantitatively characterize the Schottky barrier height, the evolution of $\Phi_{\mathrm{SB}, \mathrm{P}}$ and $\Phi_{\mathrm{SB}, \mathrm{N}}$ of GaN/graphene heterostructure as function of strain is shown in Fig. 6 . The magnitude of $\Phi_{\mathrm{SB}, \mathrm{P}}$ gradually increases and magnitude of $\Phi_{\mathrm{SB}, \mathrm{N}}$ decreases when the uniaxial strain increases to $-1 \%$, and then the magnitude of $\Phi_{\mathrm{SB}, \mathrm{P}}$ gradually decreases but magnitude of $\Phi_{\mathrm{SB}, \mathrm{N}}$ are almost not change. Consequently, the value of $\Phi_{\mathrm{SB}, \mathrm{P}}$ is negative when the uniaxial strain is $-1 \%$, where the contact type transforms from the p-type Schottky contact to ohmic contact. In contrast, with increasing the biaxial strain, the contact Schottky barrier transforms from n-type transforms from the p-type when the biaxial strain is up to $-8 \%$, and then to ohmic contact when the biaxial strain is above $0 \%$. 

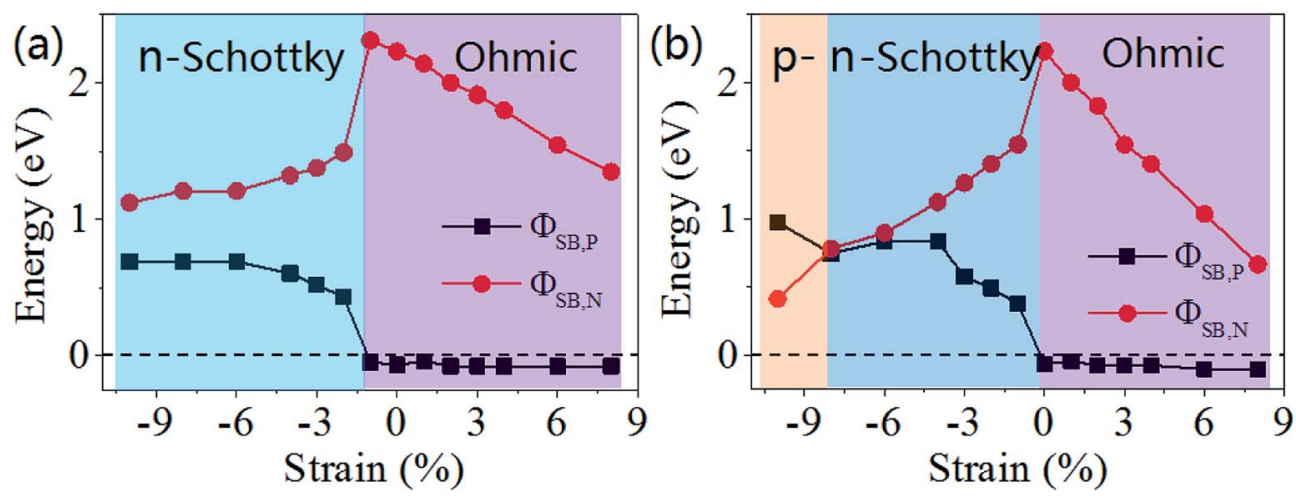

Fig. 6 Evolution of Schottky barriers of $\Phi_{\mathrm{SB}, \mathrm{P}}$ and $\Phi_{\mathrm{SB}, \mathrm{N}}$ in GaN/graphene heterostructure as a function of the applied strain: (a) uniaxial and (b) biaxial.

\section{Conclusions}

In conclusion, the structural and electronic properties of GaN/ graphene heterostructure are investigated by using firstprinciples calculation. The results showed that the electronic properties of the GaN/graphene heterostructure are well preserved owing to a weak vdW interaction between GaN and graphene. The SBH in the GaN/graphene heterostructure can be efficiently modulated by applying strain. The band gap of GaN in heterostructure decreases with increasing applied uniaxial and biaxial strains (both tensile and compressive). Moreover, the contact type transforms from the p-type Schottky contact to ohmic contact for GaN/graphene heterostructure with increasing the uniaxial strain, while the contact Schottky barrier transforms from n-type transforms to the p-type and then to ohmic contact with increasing the biaxial strain. These results suggest that graphene can be a very promising material for the metal electrode, and it can enhance the contact performance while still maintaining the electronic properties of GaN.

\section{Conflicts of interest}

There are no conflicts to declare.

\section{Acknowledgements}

This work was supported by National Natural Science Foundation of China (Grant No. 51274163), Shanxi Province Key Laboratory Research Project (Grant No. 13JS076), Key Scientific and Technological Innovation Team in Shanxi Province (Grant No. 2012KCT-25), Project of special funds for Key Discipline Project in Shanxi Province (Grant No. 2011HBSZS009).

\section{References}

1 D. Xiao, G. B. Liu, W. X. Feng, X. D. Xu and W. Yao, Phys. Rev. Lett., 2012, 108(19), 196802.

2 Z. Y. Zhu, Y. C. Cheng and U. Schwingenschlogl, Phys. Rev. B: Condens. Matter Mater. Phys., 2011, 84(15), 153402.
3 Q. L. Fang, X. M. Zhao, Y. H. Huang, K. W. Xu, T. Min and F. Ma, J. Mater. Chem. C, 2019, 7(12), 3607-3616.

4 M. L. Sun, J.-P. Chou, J. F. Gao, Y. Cheng, A. Hu, W. C. Tang and G. Zhang, ACS Omega, 2018, 3(8), 8514-8520.

5 M. L. Sun, J.-P. Chou, L. H. Shi, J. F. Gao, A. Hu, W. C. Tang and G. Zhang, ACS Omega, 2018, 3(6), 5971-5979.

6 Q. L. Fang, X. M. Zhao, Y. H. Huang, K. W. Xu, T. Min, P. K. Chu and F. Ma, Phys. Chem. Chem. Phys., 2018, 20(1), 553-561.

7 H. Shu, F. Li, C. Hu, P. Liang, D. Cao and X. Chem, Nanoscale, 2016, 8(36), 2918-2926.

8 S. K. Behera and P. Deb, RSC Adv., 2017, 7(50), 31393-31400.

9 J. E. Padilha, A. Fazzio and A. J. R. da Silva, Phys. Rev. Lett., 2015, 114(6), 066803.

10 H. C. Diaz, J. Avila, C. Y. Chen, R. Addou, M. C. Asensio and M. Batzill, Nano Lett., 2015, 15(2), 1135.

11 H. V. Phuc, N. N. Hieu, B. D. Hoi and C. V. Nguyen, Phys. Chem. Chem. Phys., 2018, 20(26), 17899-17908.

12 P. T. T. Le, N. N. Hieu, L. M. Bui, H. V. Phuc, B. D. Hoi, B. Amin and C. V. Nguyen, Phys. Chem. Chem. Phys., 2018, 20(44), 27856-27864.

13 K. D. Pham, N. N. Bieu, H. V. Phuc, I. A. Fedorov, C. A. Duque, B. Amin and C. V. Nguyen, Appl. Phys. Lett., 2018, 113(17), 171605.

$14 \mathrm{H}$. V. Phuc, V. V. Ilyasov, N. N. Hieu, B. Amin and C. V. Nguyen, J. Alloys Compd., 2018, 750(25), 765-773.

15 K. Ren, M. L. Sun, Y. Luo, S. Wang, Y. J. Xu, J. Yu and W. C. Tang, Phys. Lett. A, 2019, 383(24), 1487-1492.

16 K. Ren, M. L. Sun, Y. Luo, S. Wang, Y. J. Xu, J. Yu and W. C. Tang, Appl. Surf. Sci., 2019, 476(15), 70-75.

17 U. Kuhl and H. J. Stöckmann, Phys. Rev. Lett., 1998, 80(15), 3232-3235.

18 C. R. Dean, L. Wang, P. Maher, C. Forsythe, F. Ghahari, Y. Gao, J. Katoch, M. Ishigami, P. Moon, M. Koshino, et al., Nature, 2013, 497(7451), 598-602.

19 Q. L. Fang, Y. H. Huang, Y. P. Miao, K. W. Xu, Y. Li and F. Ma, J. Phys. Chem. C, 2017, 121(12), 6605-6613.

20 Q. L. Fang, X. M. Zhao, Y. H. Huang, K. W. Xu, T. Min, P. K. Chu and F. Ma, J. Mater. Chem. C, 2018, 6(16), 45234530. 
21 P. Rivera, K. L. Seyler, H. Yu, J. R. Schaibley, J. Yan, D. G. Mandrus, W. Yao and X. Xu, Science, 2016, 351(6274), 688-691.

22 Y. M. Li, J. Li, L. K. Shi, D. Zhang, W. Yang and K. Chang, Phys. Rev. Lett., 2015, 115(15), 166804.

23 S. Pimputkar, J. S. Speck, S. P. DenBaars and S. Nakamura, Nat. Photonics, 2009, 3(4), 180-182.

24 E. Kioupakis, P. Rinke, A. Janotti, Q. Yan and C. G. Van de Walle, Energy Conversion: Solid-State Lighting, in Computational Approaches to Energy Materials, ed. A. Walsh, A. A. Sokol and C. R. A. Catlow, John Wiley \& Sons Ltd., Oxford, U.K., 2013, pp. 231-259.

25 J. Verma, P. K. Kandaswamy, V. Protasenko, A. Verma, H. Grace Xing and D. Jena, Appl. Phys. Lett., 2013, 102(4), 041103.

26 K. Ren, S. K. Wang, Y. Luo, Y. J. Xu, M. L. Sun, J. Yu and W. C. Tang, RSC Adv., 2019, 9(9), 4816-4823.

27 Z. Y. Al Balushi, K. Wang, R. K. Ghosh, R. A. Vila, S. M. Eichfeld, J. D. Caldwell, X. Qin, Y.-C. Lin, P. A. DeSario, G. Stone, S. Subramanian, D. F. Paul, R. M. Wallace, S. Datta, J. M. Redwing and J. A. Robinson, Nat. Mater., 2016, 15(11), 1166-1171.

28 G. Kresse and J. Hafner, Phys. Rev. B: Condens. Matter Mater. Phys., 1993, 47(1), 558-561.

29 G. Kresse and J. Hafner, Phys. Rev. B: Condens. Matter Mater. Phys., 1994, 49(20), 14251-14269.

30 P. E. Blöchl, Phys. Rev. B: Condens. Matter Mater. Phys., 1994, 50(24), 17953-17979.

31 J. P. Perdew, K. Burke and M. Ernzerhof, Phys. Rev. Lett., 1996, 77(18), 3865-3868.
32 Q. Chen, H. Hu, X. J. Chen and J. L. Wang, Appl. Phys. Lett., 2011, 98(5), 053102.

33 S. Grimme, J. Comput. Chem., 2006, 27(15), 1787-1799.

34 K. Momma and F. Izumi, J. Appl. Crystallogr., 2008, 41, 653658.

35 K. S. Arunima and G. H. Richard, Appl. Phys. Lett., 2014, 105(5), 051604.

36 L. Liu, P. Jewook, A. S. Devid, F. M. Kevin, W. C. Kendal, W. Deng, B. Leonardo, C. I. Juan, A. P. Li and G. Gu, Science, 2014, 343(6167), 163-167.

37 W. Hu, T. Wang, R. Zhang and J. Yang, J. Mater. Chem. C, 2016, 4(9), 1776-1781.

38 J. E. Padilha, A. Fazzio and A. J. R. da Silva, Phys. Rev. Lett., 2015, 114(6), 066803.

39 W. Hu, T. Wang and J. Yang, J. Mater. Chem. C, 2015, 3(18), 4756-4761.

40 B. Liu, L.-J. Wu, Y.-Q. Zhao, L.-Z. Wang and M.-Q. Cai, Phys. Chem. Chem. Phys., 2016, 18(29), 19918-19925.

41 Y. Cai, G. Zhang and Y.-W. Zhang, J. Phys. Chem. C, 2015, 119(11), 13929-13936.

42 C. Xia, B. Xue, T. Wang, Y. Peng and Y. Jia, Appl. Phys. Lett., 2015, $107(19), 193107$.

43 Y. Wang and Y. Ding, Phys. Chem. Chem. Phys., 2015, 17(41), 27769-27776.

44 G. Xiao, L. L. Wang, Q. Y. Rong, H. Q. Xu and W. Z. Xiao, Phys. B, 2017, 524, 47-52.

45 J. J. Guo, Z. P. Zhou, T. X. Wang, Z. S. Lu, Z. X. Yang and C. Liu, Curr. Appl. Phys., 2017, 17(12), 1714-1720.

46 M. L. Sun, J. P. Chou, Q. Q. Ren, Y. M. Zhao, J. Yu and W. C. Tang, Appl. Phys. Lett., 2017, 110(17), 173105. 